\title{
Spontaneous retrieval of sequential non-declarative information: New software-based neuropsychological test and algorithmic implementation of cognitive dissonance principles in serial ordering
}

\author{
J uly Silveira Gomes ${ }^{1}$, Henrique Teruo Akiba ${ }^{1,2}$, Pedro Calabrez Furtado ${ }^{1}$, Raul Ando ${ }^{1,3}$, Mauri \\ Aparecido Oliveira ${ }^{1}$, Alvaro Machado Dias ${ }^{1}$ \\ 1. Federal University of São Paulo, Clinical Neuroscience Lab “LiNC". Pedro de Toledo St, $669-3^{\circ}$ floor - Vila Clementino \\ - São Paulo, Brazil. 2 Institute of Psychology of the University of São Paulo, Cidade Universitária - São Paulo, Brazil. 3 \\ Institute of Economics, State University of Campinas, Rua Pitágoras, Barão Geraldo, São Paulo, Brazil.
}

Correspondence: Alvaro Machado Dias. Address: Pedro de Toledo St, 669 - $3^{\circ}$ floor - Vila Clementino, São Paulo, SP. Brazil. ZIP Code: 04039-032.E-mail: alvaromd@usp.br, alvaromd@unifesp.br

Received: July 3, 2013

DOI : $10.5430 / j b g c . v 4 n 1 p 27$
Accepted: September 29, $2013 \quad$ Online Published: October 16, 2013

URL: http://dx.doi.org/10.5430/jbgc.v4n1p27

\section{Abstract}

Context: The principles behind the process of creating new, spontaneous sequences out of previously ordered nondeclarative stimuli have been scarcely addressed and, for such reason, remain highly unknown.

Objective: This paper has four interconnected goals: (1) introduce a new software-based neuropsychological test that can be used as a mean to assess key aspects of the way people order and reorder non-declarative stimuli, based upon cognitive dissonance principles; (2) introduce a mathematical approach to the latter in ordering/re-ordering of non-declarative stimuli; (3) assess whether the principles of cognitive dissonance in ordering/re-ordering hold for a cohort of young adults with upper socio-economic level; (4) access the extent to which the same holds for children and adolescents and trace a curve of maturation of cognitive dissonance in ordering/re-ordering.

Methods: Our multi-age and multi-language social Network Test has two stages, first subjects must order figures representing human faces in accordance with their preference; next, different pairs of figures are automatically provided and each subject is asked to fulfill the intermediate arrays that are assumed to interconnect the original pair. Our mathematical model is centered around the relation defined by increases in the distance separating these different pairs of figures in the initial order (distances 1,5 and 11) and related increases in the mean number of intermediate arrays placed in the re-ordering phase; 105 subjects were tested.

Results: The tendency to produce reorders that are consonant to the one produced in the initial phase increases with age (in other words: people feel that there are more intermediate arrays between any two individuals to which they attribute divergent affect than the contrary). This trend inspired us to propose a cognitive dissonance index in spontaneous ordering/reordering of non-declarative stimuli, which may formalize the operation of a previously unknown cognitive dimension of the human mind and may serve as an index of cognitive maturation. To the extent that further studies endorse these perspectives, the tests, formulas, and theoretical principals may support new diagnostic methods and explorations in cognitive science. 


\section{Key words}

Cognitive dissonance, Spontaneous retrieval, Maturation, Software development, Positional theories

\section{I ntroduction}

The ways by which declarative and non-declarative information is encoded and retrieved encompasses a vast field of studies, pointing toward complex interfaces between experimental data and theoretical modeling.

One perspective that has been extensively debated in the last half century and yet has not been resolved concerns the algorithmic implementation of the mechanisms that stand behind the ability to store and use positional information (herein referred to as "seriality"), associated with the encoded sequence of items ${ }^{[1,2]}$. In relation to this ability, one of the most prolific conceptions is offered by "Positional Theory" "3], which states that seriality, as encoded in the short-term memory, involves the discrete neurocomputation of the parameters related to stimuli's position.

It is as if these were in "labeled boxes" that could be manipulated according to these labels, along with the information that they carry in regard to adjacent boxes and the relative position that they set out in relation to the sequence as a whole (i.e., in the early part, near the middle; in the final portion of the sequence; for a discussion, see: Henson ${ }^{[4,5]}$ ).

Another line of thinking, presented by "Chaining Theories", is centered on the premise that each individual stimulus is encoded in association with information about (and only about) those with which it is contiguous.

This conception has become less popular in recent years due to two factors: its unsuitability for modeling the ways we deal with the association between items that are not contiguous (memory for items separated by one or more stimuli); and the difficulties of understanding how one could perform the retrieval of a part of a sequence after a particular stimulus was erroneously retrieved. Specifically, Chaining Theory is tied to equivocal predictions; e.g., that sequences following an error must incorporate it and spread it, as each subsequent step to the error would be anchored at a wrong reference point ${ }^{[6]}$.

As one may note, seriality is the key trend behind verbal ability, as it is at the core of verbal syntax ${ }^{[7,8]}$ and much has been said about the role of specific brain regions (e.g., Broca's area) in computing serial issues related to language ${ }^{[9]}$. A key perspective to bear in mind at this point is that one of the core syntactic neural computations is represented by the (compositional) capacity to find an equivalence between canonically and non-canonically ordered phrases (John loves Mary $\leftrightarrow$ Mary is loved by John).

The specific ties that verbal language establishes with high level computation in seriality may have shadowed the exploration of operational rules (algorithmic processes) in the broad field of serial ordering, especially in regard to non-declarative stimuli.

This approach is notoriously out of the scope of the original debate in non-verbal seriality, which has mainly to do with the processes behind the intentional retrieval of serial information (usually presented to the subject, who passively observes them).

As the existence of implicit rules behind freely retrieved sequences remains an open matter at the heart of one of the highest aspirations in cognitive science - the search for universal principles in information processing - it becomes suggestive that (1) this is not a matter to be resolved through the description and application of a single algorithmic process; (2) experimentally endorsed models that already target information processing in domains that originally depart from this one could be applied as part of a broad effort to understand these mechanisms. In this last sense, we believe that an interesting approach can be defined in light of the "cognitive dissonance theory", its addenda and counterparts, which 
together characterize a long history of experiments and hypotheses about how, under certain conditions, stimuli are related in the mind.

The concept of cognitive dissonance was developed by Leon Festinger ${ }^{[10]}$ to describe internal contradictions that most people try to avoid. According to the author, the possession of two mental representations and/or two goals, either leads to a reassessment of them, or the production of a third representation/intent, that enables one to resolve the conflict. The most famous experiment in support of this hypothesis was conducted by Festinger and Carlsmith ${ }^{[11]}$, where the researchers first asked a group of volunteers (students) to participate in a notoriously boring academic activity. Subsequently, participants were divided into three subgroups. The first group of subjects received an offer of $\$ 1$ to say to a supposedly unknown person (who was actually a confederate of the experimenter) that the task was exciting. The same was requested of the second group, to which was offered 20 dollars; while members of the third group were treated as "controls" (nothing was offered). At the end, all participants were asked to rate how much they liked the boring task.

The main finding was that those who received $\$ 1$ reported enjoying the task more than those who received $\$ 20$. According to the authors, doing a boring task (an activity encoded in association with a "negative valence" affective signal) to receive only $\$ 1$ produces an internally conflictive situation, which is mitigated by the revaluation of their own experience of the task, generating so a cognitive bias (attributional bias), which is less intense than in the $\$ 20$ group. The experiment of Festinger and Carlsmith ${ }^{[11]}$ became prototypical in this field and inspired dozens of other studies up until the current day.

In our view, the extent to which cognitive dissonance principles hold, the human mind can be considered to incorporate "dialectic" principles, by means of which mental stimuli associated with conflicting attributes (thesis and antithesis) converge into synthetic outputs that decrease "information entropy" and, from a certain angle, can be considered as more cost-effective than the previous state from which it emerges. Considering the philosophical insights that can be associated with this idea, it should be noted that, at the behavioral level, subjects who evaluated the aforementioned task as "not so boring" can be considered to act "irrationally", while solely regarding the mental dynamics of informational processing, they may simply express a cost-effective tendency toward consistency, simplicity and parsimony, which are the founding principles of rational thought.

In this sense, the Theory of Cognitive Dissonance can bring up important issues concerning how we encode and retrieve information and how this process produces puzzling behavioral outputs, under the aegis of two axioms: (1) We process information under the influence of a tendency to reduce entropy, regardless of the behavioral costs associated with this trend; (2) This tendency is both implicit (in the sense that people are not aware of the mental dynamics driving their behavioral biases) and spontaneous.

The assumption that we naturally oppose informational entropy and, hence, tend to favor informational consistency, can be transposed into the exploration of the algorithmic processes driving serially. This can be achieved through the development of an appropriate way to track the degree by which items that were nearer in an original serial order are defined by the subject and tend to preserve such a characteristic (and, conversely, items originally opposed tend to acquire transitive dissonant properties) in subsequent re-arrangements, and guided by unrelated principles.

In sum, we assume that a neuropsychological test is needed that can serve as a means to analyze how people deal with non-declarative information in ordering/re-ordering dynamics, and that such a test must be tied to (1) instructions assuring that ordering and re-ordering are both freely developed and not influenced by a singular organizational principle; (2) a mathematical model that can characterize the degree of consonance/dissonance between sequences.

As is noted, these perspectives advocate a broad and innovative approach to both seriality and cognitive dissonance, which radically transcends the ones that have been outlined to date in both domains. Especially significant is the fact that, despite the enormous contributions made by cognitive dissonance theory, not a single experiment was able to model the algorithmic implementation of cognitive dissonance in the human mind. In fact, it is hard to say and probably harder to 
believe that somebody has not ever considered the possibility of treating cognitive dissonance as a broad principle related to informational entropy.

First, all experiments described in indexed publications involve manipulations of motivation (through rewards and punishments), which reduce the potential scope of the principle to motivational contexts. Second, the canonical approach to cognitive dissonance (as presented in the vast majority of the hundreds of publications on the theme) revolves around establishing whether dissonance can or cannot be found in a particular context. The design that we bear in mind aims to establish various degrees of dissonance/consonance in the process of re-arranging previously ordered non-declarative stimuli, in contexts where there is no motivational bias; that is, where the intention to re-edit parts of the original sequence is deemed irrelevant (non-motivated context).

In our proposal, the quest for implicit rules in spontaneous ordering/re-ordering of non-declarative information is tied to new investigations into cognitive dissonance, by which we face the challenge of quantifying the degree by which different people (and groups) tend to avoid dissonance, in a context that favors the proposition of an "index of cognitive dissonance in the human mind" to speak about such differences in performance.

\section{Objectives}

This study has four interrelated goals:

1) Introduce a new software-based neuropsychological test that can be used as a mean to assess key aspects of the way people order and re-order non-declarative stimuli, in accordance with the above principles (no external rewards; different guiding principles to ordering and re-ordering). As we will show, this same test is ready to be used to access a specific type of sequential memory test, related to the capacity to retrieve non-declarative, social stimuli.

2) In line with the above objective, we propose an original, straightforward mathematical approach to the sequences that leads to the definition of a cognitive dissonance index in ordering/re-ordering of non-declarative stimuli (cognitive dissonance as a broad principle of information processing in the human mind).

3) Assess whether the broad and reward/punishment-independent principles of cognitive dissonance in ordering/reordering hold for a cohort of young adults with upper socio-economic level and no records of psychiatric or neurological disorder, using the new test.

4) Access the extent to which the same holds for children 8 years-11 years old and adolescents 12 years-16 years old and to explore the possibility of delineating a curve of maturation of cognitive dissonance as a broad principle of information processing in the human mind. Methods and results will be presented together in the next section, since our main results - the test and the mathematical approach - are methodological in their nature.

\section{Methods and results}

\subsection{The new social network test software}

The new test has two versions, one for children and one for adults. Both of them are freely-distributed internet-based software (link), which can be used online or offline (see Figure 1). Results can be automatically uploaded to a database, where basic R-based statistical packages are implemented, in order to speed-up analyses and stimulate use.

The test involves twelve sex-balanced public-domain images of human faces. Race was also balanced in an effort to represent a random sample of members of an urban Western society. In the adult version, the ages of the photographed people were estimated by a group of four evaluators as ranging from 20 years to 70 years, whereas the children are $\approx 7$ 
years-10 years old (see Figure 2). When used for cognitive dissonance in ordering/re-ordering, links for the adult version are snt.oda.mat.br (Portuguese) and snt.oda.mat.br/en (English), while links for the child version are sntk.oda.mat.br and sntk.oda.mat.br/en. When used as a serial memory (a "social serial ordering memory test"), the links for the adult version are smt.oda.mat.br and smt.oda.mat.br/en. There are no child versions available for serial memory at the moment.

Figure 1. Phases 1 and 2 of our test (In the former, the figures are spread on the screen, while in the latter, they are displayed in a side bar.)

Figure 2. Child version of our test. The subject filled the links for the $(2,7)$ pair, using Figure 3 and 6

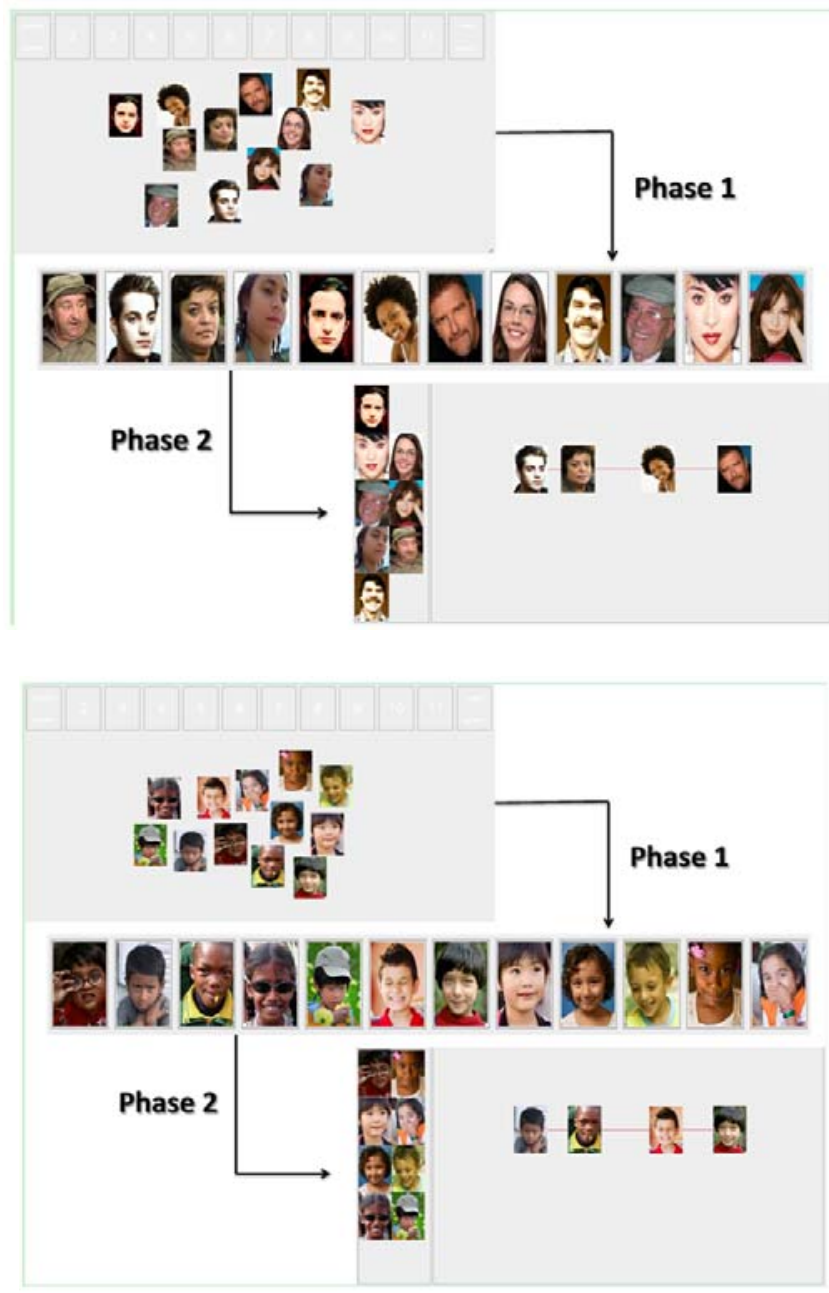

Either when used as a serial ordering memory test or as a mean to evaluate principles of spontaneous ordering/re-ordering, the test is divided into two parts: first, the subjects are asked to lineup the images in order of preference, starting from the one they liked the least. The criterion of choice for ordination remains free from any interference (as explained below, our survey suggested that most people tend to use "sympathy" as the main criterion). After lining up the sequence, the subjects are then asked to press the "I am satisfied" button and the sequence would vanish and new instructions would appear.

These new instructions state that they must use as many of the remaining figures as they wish to represent the links (the social network) that they believe to properly intermediate the pairs of images that will be given next. That is, each of the remaining "people" can be potentially responsible for intermediating the given pair and the task is basically centered on the definition of who should be included and who should not.

These pre-set pairs are represented by images of faces that have occupied positions $(2,7)(4,5)(6,11)(1,12)$ and $(8,9)$ in the original ordering by preference. As one can see, the distance separating the pairs $(4,5)$ and $(8,9)$ is 1 , the distance separating the pairs $(2,7)$ and $(6,11)$ is 5 , and the distance of the pair $(1,12)$ is 11 ; and, as one may guess, the key variable in the test is the way people deal with these distances. The child version follows the same pattern. Figure 2 presents a sequence made by a subject for pair $(2,7)$. 


\subsection{Cognitive dissonance index in ordering/ re-ordering of non-declarative stimuli}

The first step to the creation of the cognitive index formula was to average the number of intermediate links (steps) in re-ordering (phase 2), for the adult group $(\mathrm{N}=35$ ), using distances D1, D5 and D11 (see Figure 3). By that means, it was possible to observe that, in general, the number of intermediate links tend to increase with distance, regardless of the fact that order and re-ordering are not conceptually related.

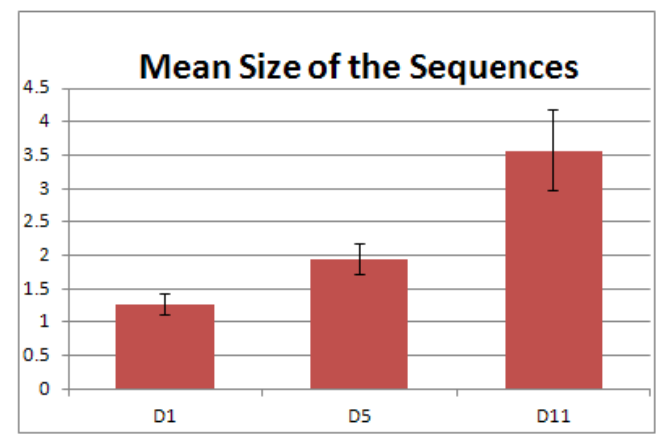

Figure 3. Average number of intermediate links in re-ordering sequences

After the establishment of such a principle, we used the method of least squares to adjust a family of functions to points $(0$, $0),(1, \mathrm{D} 1),(5, \mathrm{D} 5)$. We called this function $\varphi$, such as $\varphi=6-\exp \left(a x^{3}+b x^{2}+c x+d\right)$. This function was chosen due to two characteristics: it warrants that $\varphi$ will always be less than or equal to six and that it passes through points $(0,0)$, $(1$, D1), (5, D5). The chosen function was limited to six links due to previous research on the famous six degrees of separation paradigm (regardless of the distance, the interpolation of more than six figures was neither expected nor observed), whereas the necessity to pass through these points simply reflects our predefined norms.

As one may note, $\varphi$ allows the determination of the optimal number of links between a given pair $\mathrm{i}, \mathrm{j}$ with distance $j-i$. Is it possible, considering the number of links actually placed, $L_{i, j}$, to define scores by $\varphi(j-i)-L_{i, j}$. A given score must be always positive and normalized by the maximum possible distance, which is given by the maximum between $10-\varphi(j-i)$ and $\varphi(j-i)$. By doing this, in practice we have defined that score values will always range from 0 to 1 , with 0 being the optimal value and 1 the worst value. Finally, we considered that, since values increase as performance decreases, the final result should be subtracted from 1 , so that 0 becomes the worst value and 1 the optimal value. Figure 4 presents the curve that was rendered by these procedures.

Figure 4. The "consonance curve" according to the principles that we have developed. Axis $y$ plots the number of expected links between the images with the distances presented in Axis x.

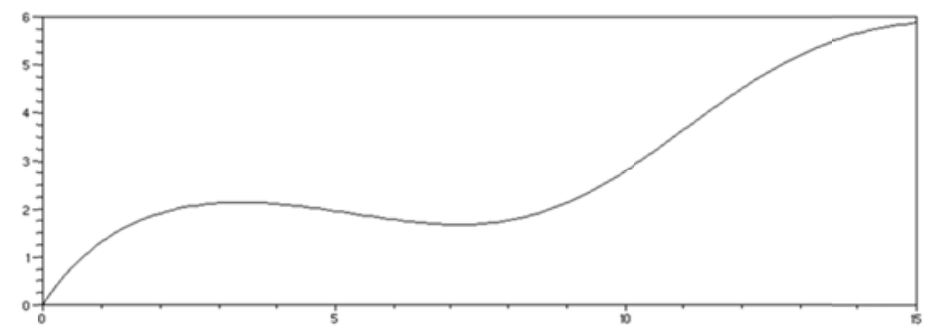

Once we defined a way to analyze performance in terms of the number of intermediate links for a given pair (D1, D5, D11), we focused on the evaluation of the extent to which the principles of consonance hold along these intermediate links. That is, whether the organization of the internal sequence preserves that of the original sequence to any extent. To treat this problem, the critical variable is the number of permutations that are necessary to put the images in order; for instance, suppose that in the re-ordering phase, someone proposes that the sequence $3,6,5,7$ intermediate the pair $(1,12)$; as one may note, it will be necessary to switch the position of a single pair (6-5 to 5-6), in order to order them. Now, supposing that the sequence was $7,3,6,5$, there would be the necessity to switch two pairs. 
In practice, this problem introduces a "Kendall tau index", dedicated to the evaluation of the internal organization of the sequences, based upon the idea of determining the number of permutations needed to transform an actual sequence into a maximally linear one; as following:

$$
\Omega=\frac{\frac{C-D}{\frac{1}{2} n(n-1)}+1}{2}
$$

This formula reads: Consonant items (the ones that are in the right place) minus dissonant items (the ones that have to be replaced), divided by half of the number of arrays, times the number of arrays minus 1 . Subsequently, the tau coefficient was normalized to the interval $[0,1]$, as following:

$$
\tau=\frac{C-D}{\frac{1}{2} n(n-1)}
$$

Finally, the "Index of Cognitive Dissonance of the Human Mind" - "I" was determined as the sum of the coefficients $\Omega_{i j}$ weighted by the normalized difference between the number of steps and the value of $\varphi$.

$$
I=\sum \Omega_{i j}\left(1-\frac{\left|L_{i, j}-\varphi(j-i)\right|}{\max \{\varphi(j-i), 10-\varphi(j-i)\}}\right)
$$

\subsection{Performance of an adult cohort ( 20 years to 35 years old)}

For adults, it was revealed that people generally preserved a consonant principle in ordering/re-ordering of the sequences, as one can see in Table 1.

Table 1. Data obtained from 35 adults (20 years to 35 years old) in ordering/re-ordering

\begin{tabular}{lllll}
\hline Data & Mean & Variance & St. D. & Conf. I. \\
\hline All & 3.456 & 0.197 & 0.444 & 0.135 \\
$4-5$ & 0.931 & 0.852 & 0.923 & 0.281 \\
$8-9$ & 1.344 & 1.09 & 1.044 & 0.319 \\
$2-7$ & 1.862 & 1.837 & 1.355 & 0.414 \\
$6-11$ & 2.034 & 2.320 & 1.523 & 0.465 \\
$1-12$ & 3.551 & 3.756 & 1.938 & 0.592 \\
\hline
\end{tabular}

The overall performance of the 35 adults that took part in the experiment is also presented in Figure 5. This graph shows the calculated "I" (Index of Cognitive Dissonance of the Human Mind) for each of the pre-defined pairs.

Figure 5. Overall performance of the 35 adults (20 years to 35 years) in relation to "I" (Index of Cognitive Dissonance of the Human Mind)

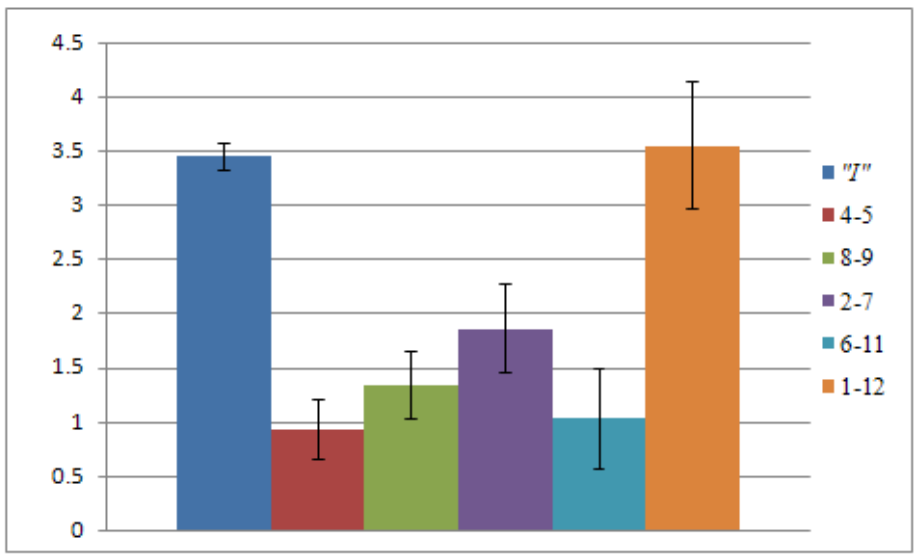




\subsection{Performance of a young cohort, involving children ( 8 years to 11 years old) and teenagers ( 12 years to 16 years old); defining the curve of maturation of I}

In this section we explored: (1) whether cognitive dissonance would also hold (and to what extent) in younger cohorts of normal individuals; (2) the possibility of defining the curve of maturation of cognitive dissonance. To achieve such goals, we selected two groups ( $\mathrm{N}=35$ for each), one consisted of children, with ages ranging from 8 years to 11 years old; and one consisted of teenagers, with ages ranging from 12 years to 16 years old. Below we present the results found with regard to the younger group (see Table 2).

Table 2. Data obtained from children (8 years to 11 years old) in ordering/re-ordering

\begin{tabular}{lllll}
\hline Data & Mean & Variance & St. D. & Conf. I. \\
\hline Score & 3.418 & 0.106 & 0.326 & 0.097 \\
$4-5$ & 1.033 & 0.998 & 0.999 & 0.300 \\
$8-9$ & 0.800 & 0.993 & 0.996 & 0.299 \\
$2-7$ & 1.066 & 0.822 & 0.907 & 0.272 \\
$6-11$ & 1.033 & 0.412 & 1.188 & 0.356 \\
$1-12$ & 0.933 & 1.098 & 1.048 & 0.315 \\
\hline
\end{tabular}

Figure 6. Overall performance of the children $(8$ years to 11 years) in relation to "I" (Index of Cognitive Dissonance of the Human Mind)

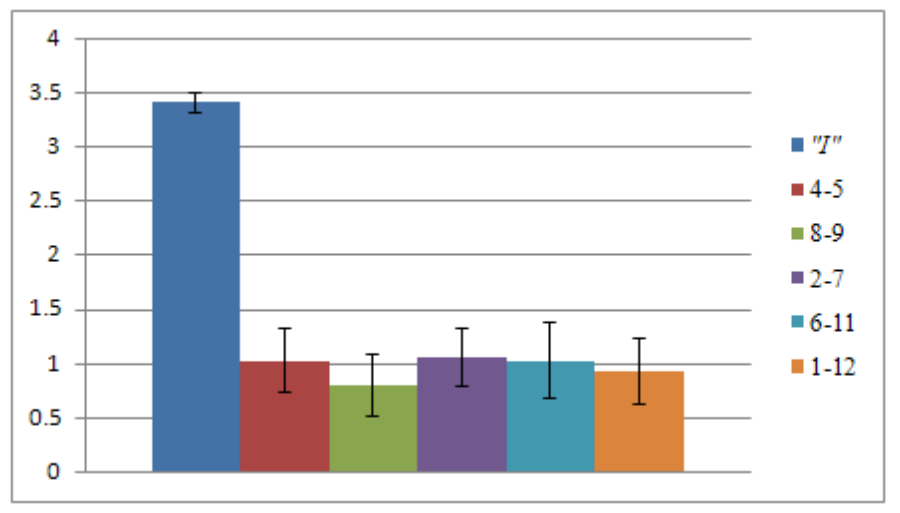

As it stems from a comparison of Figure 5 and Figure 6, there are striking differences between adult and child spontaneous avoidance of cognitive dissonance in serial ordering of human-like stimuli.

In Table 3, we present the results for the adolescent cohort.

Table 3. Data obtained from teenagers (12 years to 16 years old) in ordering/re-ordering

\begin{tabular}{lllll}
\hline Data & Mean & Variance & St. D. & Conf. I. \\
\hline Score & 3.676 & 0.116 & 0.341 & 0.128 \\
$4-5$ & 0.736 & 0.760 & 0.871 & 0.329 \\
$8-9$ & 0.631 & 0.578 & 0.766 & 0.287 \\
$2-7$ & 1.526 & 0.707 & 0.841 & 0.317 \\
$6-11$ & 1.684 & 1.228 & 1.108 & 0.418 \\
$1-12$ & 2.368 & 2.134 & 1.460 & 0.551 \\
\hline
\end{tabular}

As suggested by a comparison of Figure 6 and Figure 7, the principle holds better for adolescents than for children although it holds even more efficiently for adults. Results are presented on the Table 4 below. 
Figure 7. Overall performance of teenagers $(12$ years to 16 years) in relation to "I" (Index of Cognitive Dissonance of the Human Mind)

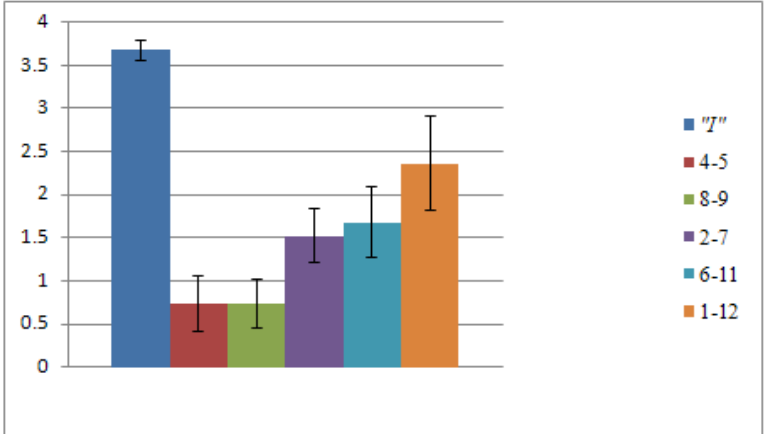

Considering a level of statistical significance of 95\%, the results using Exact Test showed significant differences between groups for pair 8-9 $\left(\chi^{2}=9.354\right.$ and $p$-value $\left.=.008\right)$; pair 2-7 $\left(\chi^{2}=8.504\right.$ and $p$-value $\left.=.013\right)$; pair 6-11 $\left(\chi^{2}=11.607\right.$ and $p$-value $=.002)$ and pair $1-12\left(\chi^{2}=38.317\right.$ and $p$-value $\left.<.001\right)$. No statistical differences were found between groups for pair $4-5\left(\chi^{2}=.134\right.$ and $p$-value $\left.=.936\right)$.

Bearing that in mind, we approached our last goal, which was the establishment of the curve of maturation of the tendency. These results are plotted in Figure 8.

Table 4. Test statistics ${ }^{*} \uparrow$

\begin{tabular}{llllll}
\hline & Pair 4-5 & Pair 8-9 & Pair 2-7 & Pair 6-11 & Pair 1-12 \\
\hline Chi-Square & .134 & 9.354 & 8.504 & 11.607 & 38.317 \\
df & 2 & 2 & 2 & 2 & 2 \\
Asymp. Sig. & .935 & .009 & .014 & .003 & .000 \\
Exact Sig. & .936 & .008 & .013 & .002 & .000 \\
Point Probability & .000 & .000 & .000 & .000 & .000 \\
\hline
\end{tabular}

Note. ${ }^{*}$. Kruskal Wallis Test; ${ }^{\dagger}$. Grouping Variable Group

Figure 8. Overall performance of the three experimental groups: adults, children and teenagers in relation to "I" (Index of Cognitive Dissonance of the Human Mind).

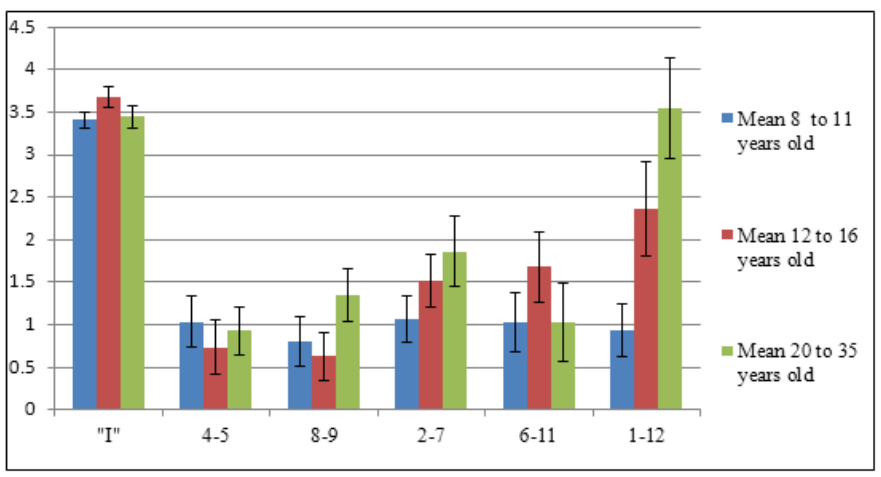

As it integrates results for all three groups, this figure provides us with an opportunity to consider whether the avoidance of cognitive dissonance in serial ordering might be a developmental trait.

\section{Discussion}

As shown by our results, adults have a strong tendency to avoid dissonance, whereas children and teenagers do not respond in the same way (see Figure 8). In practical terms, this phenomenon occurs primarily because adults tend to be more consistent in the tendency to increase the number of intermediate links, while increasing the distance from the starting pair - although it is also true that, to a certain extent, they also tend to preserve the internal organization of the sequences (intermediate links). 
There may be several reasons behind this phenomenon, but we speculate that principles of executive functioning play a prominent role among them. From this perspective, the maturation of a tendency to treat information and behavior in accordance with objective, pre-established goals involves general organization principles as broad and as basic as the ones that we have described here. Unfortunately, we cannot be sure about that: more robust studies are needed.

\section{Conclusion}

In this study, we began with a focus on spontaneous ordering/re-ordering of non-declarative information. To grasp the principles governing such behavior, we introduced a new conception of cognitive dissonance, a software-based neuropsychological task that can be used to test it (in two versions), a formula for the Consonance Index (I) and experimental results of the application of the method in children, adolescents, and adults.

This study suggests the existence of a general trend toward the avoidance of dissonant organization in spontaneous ordering/re-ordering of non-declarative information, which we believe may extend to other types of stimuli.

If this proves to be the case, we will be faced with a new dimension of human cognition, which may be explored in several senses, including the creation of new psychological and psychiatric batteries.

\section{Conflict of interests}

None declared.

\section{References}

[1] Glanzer, M., Cunitz, A. R. Two storage mechanisms in free recall. Journal of Verbal Learning and Verbal Behavior. 1966; 5(4): 351-360. http://dx.doi.org/10.1016/S0022-5371(66)80044-0

[2] Rundus, D. Analysis of rehearsal processes in free recall. Journal of Experimental Psychology. 1971; 89(1): 63-77. http://dx.doi.org/10.1037/h0031185

[3] Conrad, R. Order error in immediate recall of sequences. Journal of Verbal Learning and Verbal Behavior. 1965; 4(3): 161-169. http://dx.doi.org/10.1016/S0022-5371(65)80015-9

[4] Henson, R. N. A. Unchained Memory: Error Patterns Rule out Chaining Models of Immediate Serial Recall. The Quarterly Journal of Experimental Psychology Section A: Human Experimental Psychology. 1996; 49(1): 80-115.

[5] Henson, R. N. A. Short-Term Memory for Serial Order: The Start-End Model. Cognitive Psychology. 1998; 36(2): 73-137. doi: http://dx.doi.org/10.1006/cogp.1998.0685

[6] Henson, R. Serial order in short-term memory. The Psychologist. 2001; 14(2): 70-73.

[7] Ben-Shachar, M., Hendler, T., Kahn, I., Ben-Bashat, D., Grodzinsky, Y. The Neural Reality of Syntactic Transformations. Psychological Science. 2003; 14(5): 433-440. http://dx.doi.org/10.1111/1467-9280.01459

[8] Ben-Shachar, M., Palti, D., Grodzinsky, Y. Neural correlates of syntactic movement: converging evidence from two fMRI experiments. Neuroimage. 2004; 21(4): 1320-1336. http://dx.doi.org/10.1016/j.neuroimage.2003.11.027

[9] Fedorenko, E., Behr, M. K., Kanwisher, N. (2011). Functional specificity for high-level linguistic processing in the human brain. Proceedings of the National Academy of Sciences. http://dx.doi.org/10.1073/pnas.1112937108

[10] Festinger, L. (1957). A theory of cognitive dissonance. Stanford: Stanford University Press.

[11] Festinger, L., Carlsmith, J. (1959). Cognitive consequences of forced compliance. Journal of abnormal and social psychology, 58(2): 203-210. http://dx.doi.org/10.1037/h0041593 